\title{
The Potential of Photon Activation and Neutron Activation Techniques for Fast Soil Characterization
}

\author{
$\underline{\text { Adrien Sari }}$ \\ CEA List \\ Gif-sur-Yvette, France \\ adrien.sari@cea.fr \\ Frédérick Carrel \\ CEA List \\ Gif-sur-Yvette, France \\ frederick.carrel@cea.fr \\ Nicolas Dufour \\ CEA List \\ Gif-sur-Yvette, France \\ nicolas.dufour3@cea.fr
}

Lucile Mondon

VINCI Construction Terrassement

Nanterre, France

lucile.mondon@vinci-construction.com

\author{
Sara Garti \\ CEA List \\ Gif-sur-Yvette, France \\ sara.garti@cea.fr \\ Jonathan Dumazert \\ CEA List \\ Gif-sur-Yvette, France \\ jonathan.dumazert@cea.fr
}

Caroline Mougel

CEA DEN
Gif-sur-Yvette, France

caroline.mougel@cea.fr

Laurent Boutillon

VINCI Construction Grands Projets

Rueil-Malmaison, France

laurent.boutillon@vinci-

construction.com

\author{
Frédéric Lainé \\ CEA List
}

Gif-sur-Yvette, France

frederic.laine@cea.fr

Hamid Makil

CEA List

Gif-sur-Yvette, France

hamid.makil@cea.fr

$$
\begin{aligned}
& \text { Adeline Masset } \\
& \text { CEA DEN }
\end{aligned}
$$

Gif-sur-Yvette, France adeline.masset@cea.fr

\author{
Jean-Pierre Lejeune \\ VINCI Construction Grands Projets \\ Rueil-Malmaison, France \\ jean-pierre.lejeune@vinci- \\ construction.com
}

Abstract-In the frame of a partnership between CEA and VINCI, various measurement techniques are applied to soil analysis and tested in different laboratories located at CEA Saclay (France). This paper deals with two nuclear measurement techniques assessed in this project. More specifically, this paper presents the feasibility study carried out for two non-destructive active methods: photon activation and neutron activation. First, some atomic nuclides are activated either by photons or neutrons. Secondly, gamma-rays of specific energies are emitted by activated nuclides and gamma-ray spectrometry enables to identify these activated nuclides. Calibration of the full measurement system with reference samples would enable to quantify the mass of activated nuclides. Irradiations performed for photon activation measurements were conducted using a linear electron accelerator (linac) as the latter enables to generate high-energy photons by Bremsstrahlung thanks to its conversion target. Furthermore, irradiations performed for neutron activation measurements were also conducted with a linac. Indeed, photons may be converted to neutrons by photonuclear reactions using a secondary target. In the frame of this project, experiments were carried out at the SAPHIR platform (CEA Saclay) with a Linatron-M9 VARIAN linac. The electron energy was either 6 or $9 \mathrm{MeV}$. For neutron activation measurements, a secondary target made of heavy water has been used as neutron source and a polyethylene cell enabled to thermalize neutrons and increase the number of reactions of interest. In this paper, we present the different experimental setups and the measurement protocols established for this feasibility study. We show experimental results obtained with raw material samples coming from three construction sites.

Keywords-photon activation, neutron activation, linear electron accelerator (linac), gamma spectrometry, prompt gammarays, delayed gamma-rays, soil characterization.

\section{INTRODUCTION}

In the frame of a partnership between CEA and VINCI, various measurement techniques are applied to soil analysis and tested in different laboratories located at CEA Saclay (France). This paper deals with two nuclear measurement techniques assessed in this project. More specifically, this paper presents the feasibility study carried out for two nuclear non-destructive active methods: photon activation and neutron activation [1-3]. First, some atomic nuclides are activated either by photons or neutrons. Secondly, gamma-rays of specific energies are emitted by activated nuclides and gamma-ray spectrometry enables to identify these activated nuclides. In summary, the aims of this study are the following:

- Assessment of the potential of photon activation and neutron activation for fast soil analysis;

- Implementation using an HPGe detector and a linear electron accelerator (linac) operated at either 6 or $9 \mathrm{MeV}$;

- Tests on samples of raw material coming from three different construction sites.

The Linatron ${ }^{\circledR}$ M9 manufactured by VARIAN (now Varex Imaging) and located at the SAPHIR platform (CEA Saclay) was used for these experiments (shown in Fig. 1). This linac generates either 6 or $9 \mathrm{MeV}$ electrons. High-energy photons are produced by Bremsstrahlung in its conversion target.

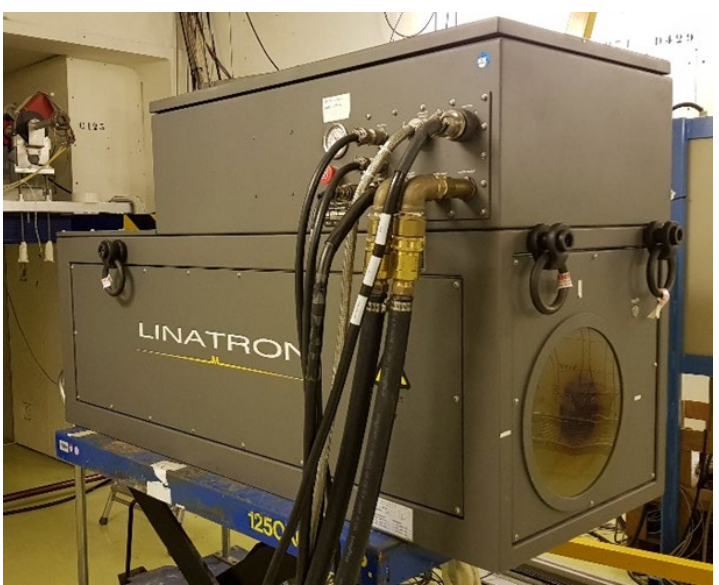

Fig. 1. The Linatron-M9 linac housed at the SAPHIR platform (CEA Saclay, France). 


\section{PhOtON INTERROGATION}

Photon activation measurements are illustrated by Figs. 2 and 3 and were carried out with the following measurement protocol:

- Electron energy: $9 \mathrm{MeV}$;

- Pulse frequency: $200 \mathrm{~Hz}$;

- Irradiation time: $10 \mathrm{~min}$;

- Cooling time: 2 min;

- Acquisition time: $10 \mathrm{~min}$.

The gamma-ray spectrum of the background is shown in Fig. 4. The latter is due to the natural radioactivity and to the history of the SAPHIR platform. Indeed, presence of ${ }^{137} \mathrm{Cs}$ is explained by the fact that the building used to house a research reactor a few decades ago. Fig. 5 presents the delayed gammaray spectrum obtained for the sample No. 1. Two radioactive nuclei seem to be produced by photon activation at $9 \mathrm{MeV}$ : ${ }^{56} \mathrm{Fe}^{*}$ and ${ }^{28} \mathrm{Si}^{*}$. Using a $30 \mathrm{MeV}$ linac, much more elements would be identified in samples by photon activation [4]. However, deployment of such a linac would not be permitted on a construction site.

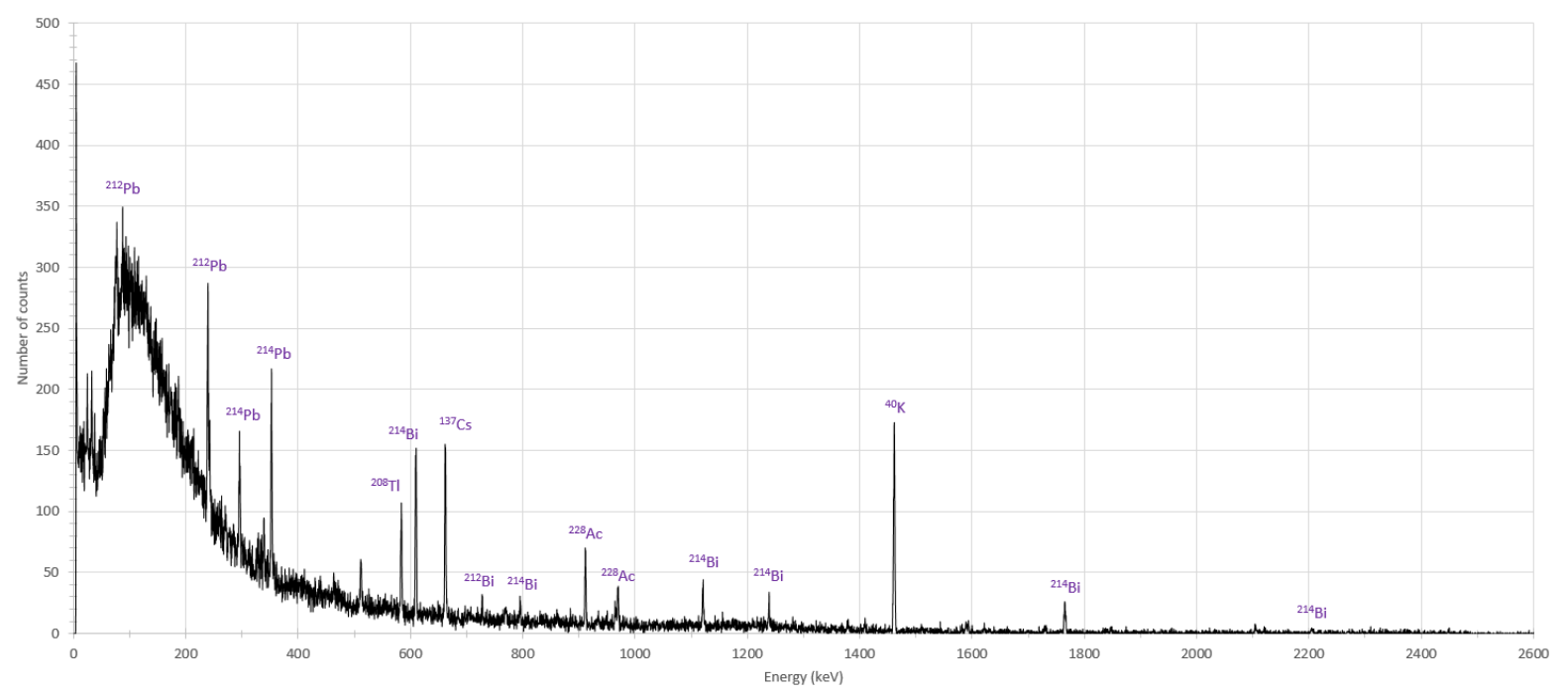

Fig. 4. Delayed gamma-ray spectrum of the background.

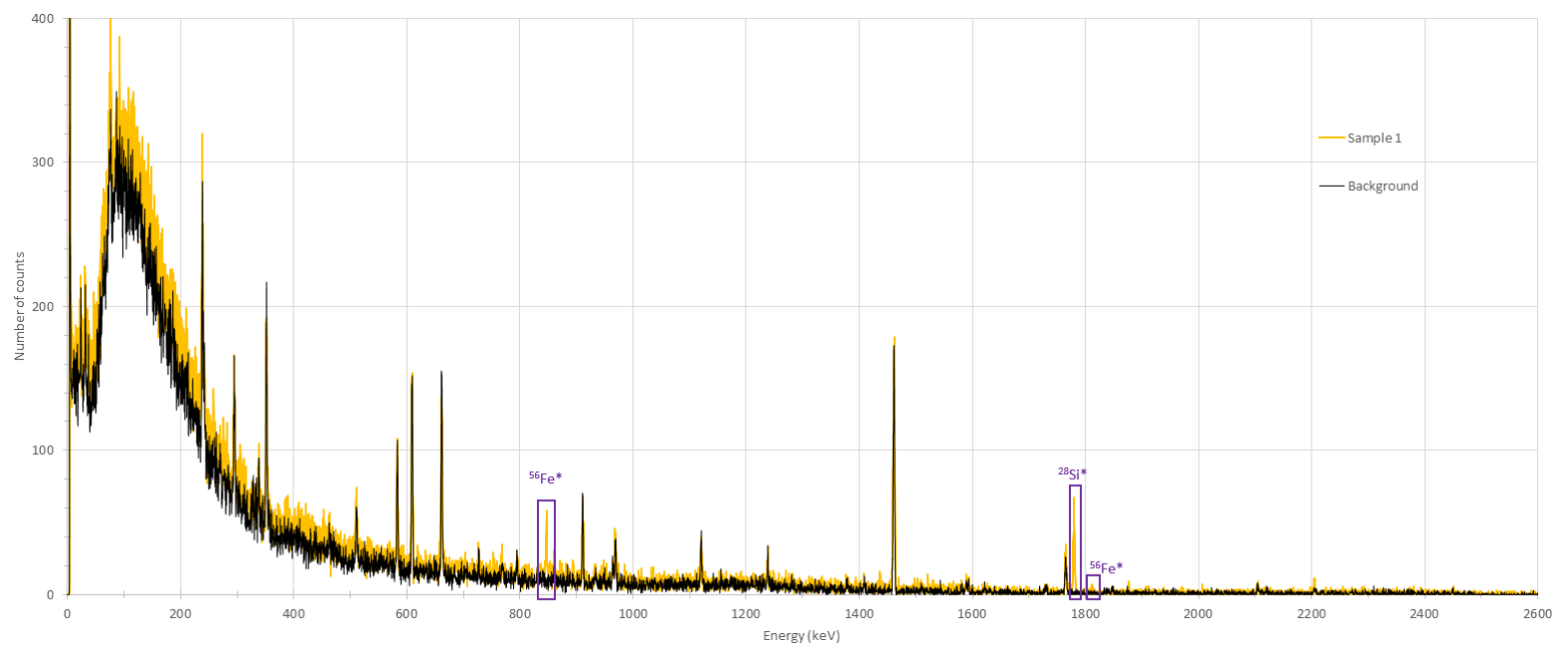

Fig. 5. Delayed gamma-ray spectrum of the sample No. 1.

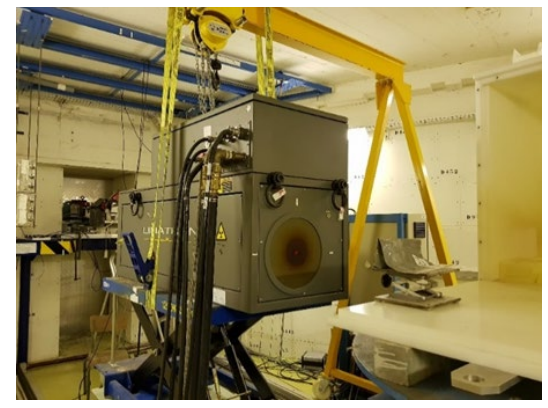

Fig. 2. Photon activation measurement: irradiation step.

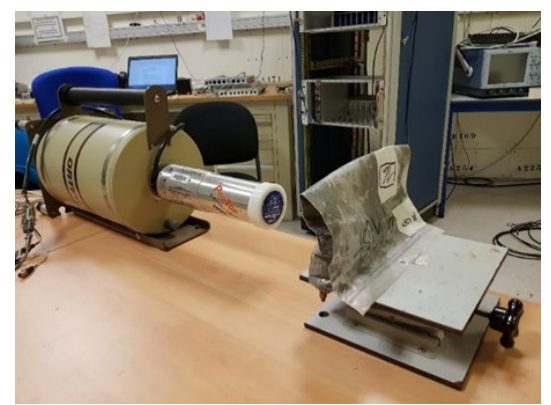

Fig. 3. Photon activation measurement: delayed gamma-ray detection step. 


\section{NEUTRON INTERROGATION}

\section{A. Production of photoneutrons}

Photoneutrons are produced by $(\gamma, n)$ reactions on deuterium using a secondary target made of heavy water $\left(\mathrm{D}_{2} \mathrm{O}\right)$, as shown in Fig. 6. The electron energy was set at $9 \mathrm{MeV}$ and the mass of heavy water was $16 \mathrm{~kg}$. At $200 \mathrm{~Hz}$, the average emission intensity is $1.16 \times 10^{10}$ neutrons per second in $4 \pi$ steradians [5]. Fast photoneutrons (energy spectrum shown in Fig. 7 [5]) are thermalized thanks to a polyethylene cell [6].

\section{B. Detection of delayed gamma-rays: setup No. 1}

The first experimental protocol dedicated to the detection of delayed gamma-rays was the following:

- $\quad$ Linac operated at a frequency of $200 \mathrm{~Hz}$;

- 20 min of irradiation time;

- 2 min of cooling time;

- 20 min of acquisition time.

Fig. 8 shows both a sample of soil placed in a corner of the neutron cell (ouside the photon beam) and a sample of soil placed in front of the HPGe detector. Fig. 9 presents experimental results obtained. Titanium, manganese, magnesium, sodium, vanadium, and aluminum are detected.

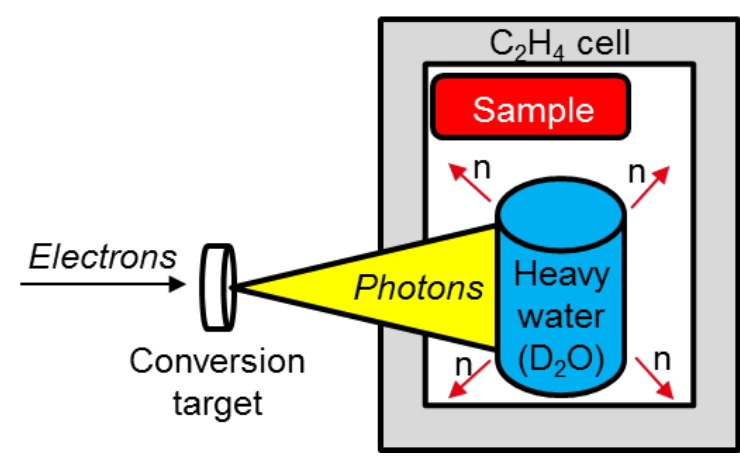

Fig. 6. Neutron activation measurement: production of photoneutrons.

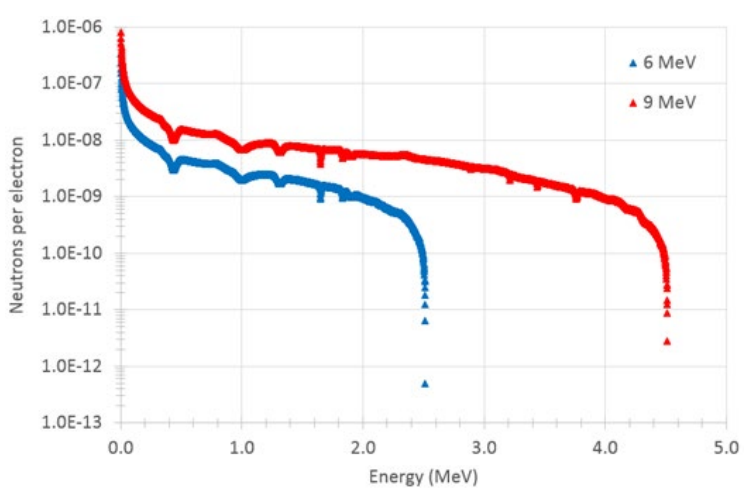

Fig. 7. Neutron energy spectra for two electron energies (MCNP6 simulation results) [5].

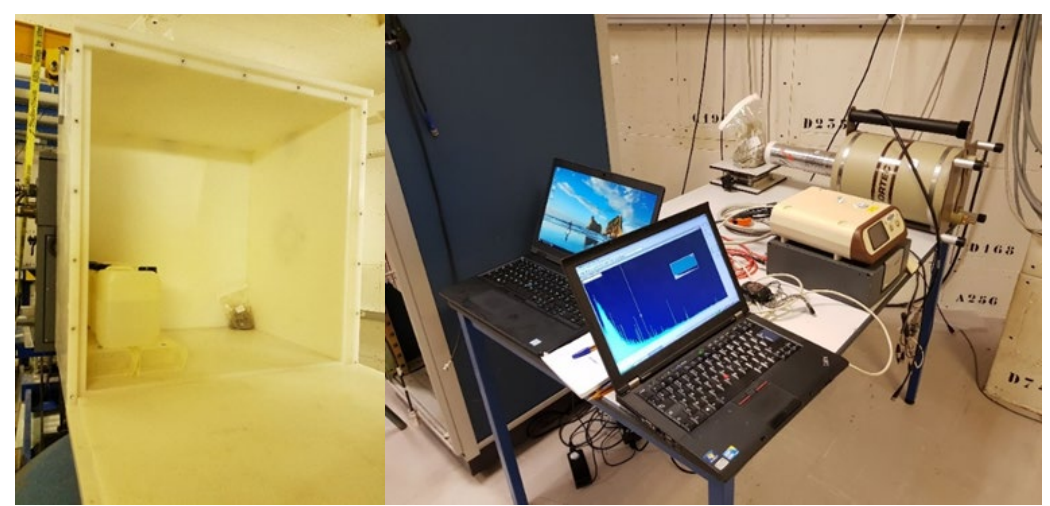

Fig. 8. On the left: irradiation step (neutron cell in open position). On the right: gamma-ray spectrometry detection step with an HPGe detector.

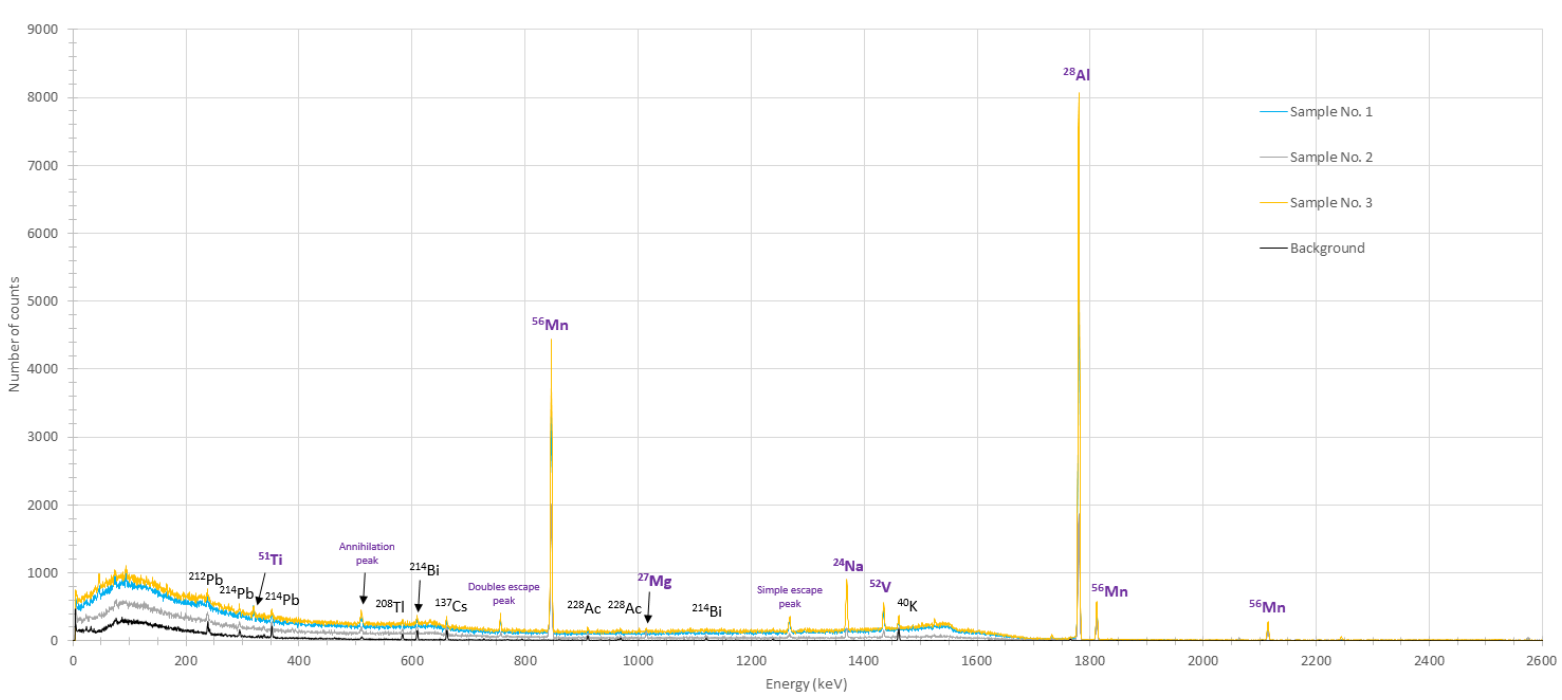

Fig. 9. Delayed gamma-ray spectra obtained for the three samples of soil with setup No. 1. 


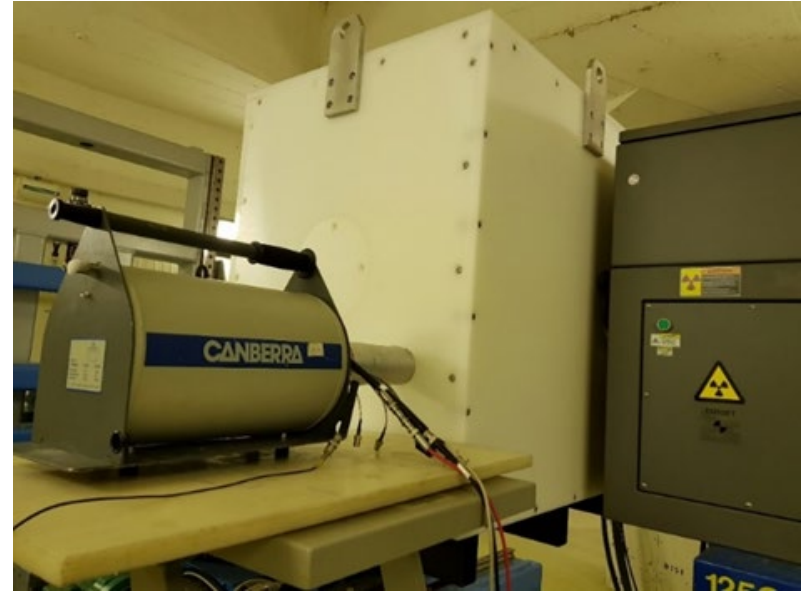

Fig. 10. Neutron activation measurement: view of the setup No. 2. HPGe detector placed on the side of the neutron cell.

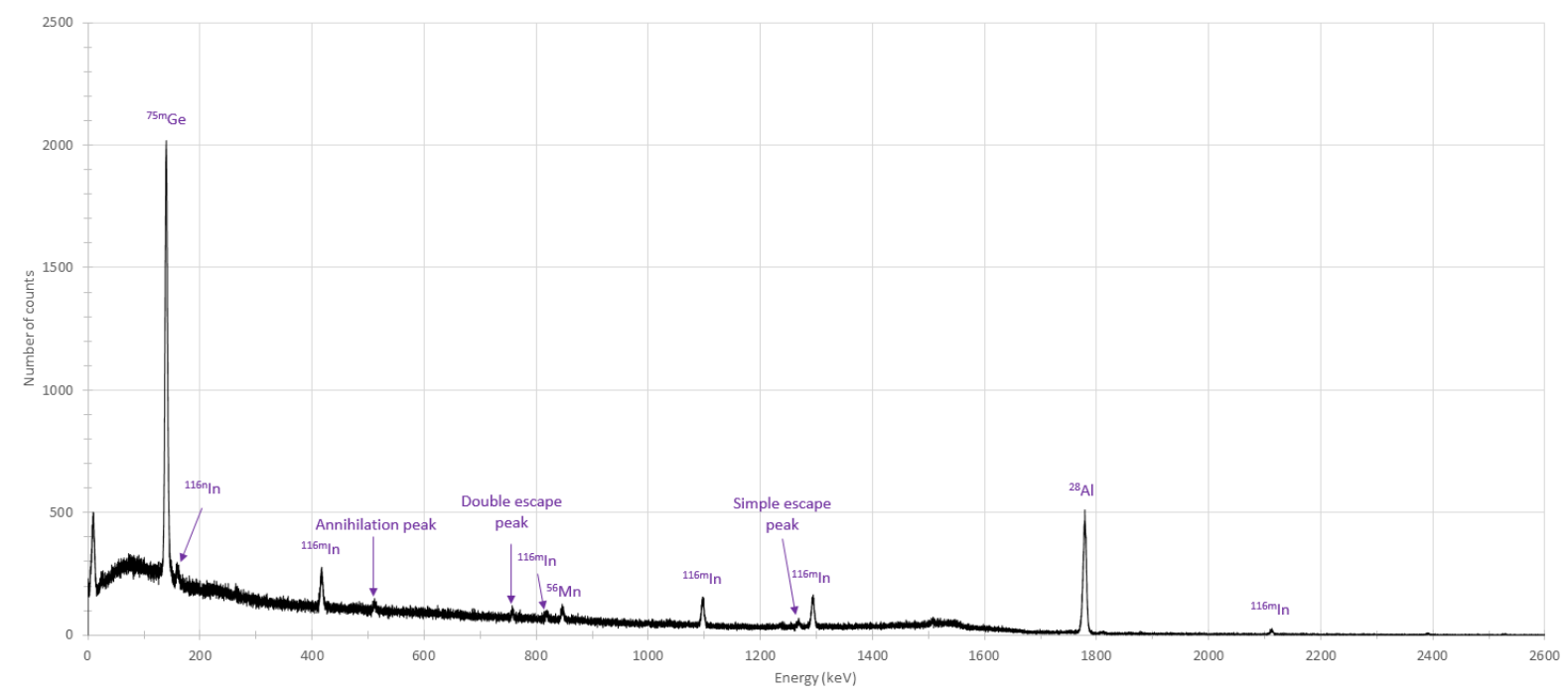

Fig. 11. Delayed gamma-ray spectrum of the background obtained with the setup No. 2.

\section{Detection of delayed gamma-rays: setup No. 2}

A second setup (shown in Fig. 10), more adapted to the detection of delayed gamma-rays emitted by radionuclides characterized by short half-lives, has been tested. The experimental protocol was the following:

- Linac operated at $6 \mathrm{MeV}$ and $40 \mathrm{~Hz}$;

- $16 \mathrm{~kg}$ of heavy water $\left(1.62 \times 10^{9}\right.$ neutrons per second in $4 \pi \mathrm{sr}[5])$;

- 5 min of irradiation time;

- Gamma spectrum acquisition launched immediatly once linac switched off.

The delayed gamma-ray spectrum of the background is shown in Fig. 11. This spectrum shows the feasibility of such measurement (HPGe detector not degraded) but a shielding around the HPGe detector would be required to reduce activation of the detector itself [7] and thus the background.

\section{Detection of prompt gamma-rays}

Measurements aiming at detecting prompt gamma-rays from neutron activation between linac pulses have been explored. Indeed, as half-life of neutrons in the cell is on the order of $1 \mathrm{~ms}$, neutron activation reactions occur after a few hundreds of microseconds up to a few milliseconds after the gamma flash from the linac. Prompt gamma-rays from neutron activation reactions are then emitted after the gamma flash and can be potentially detected.

Detection of prompt gamma-rays, which is complementary to the detection of delayed gamma-rays, is a technical challenge. Measurement of gamma-rays is carried out between linac pulses. The linac and the HPGe detector have to be synchronized. The HPGe detector has to be inhibited during the gamma flash (risk of saturation of the detector), and the detector must recover quite fast. 


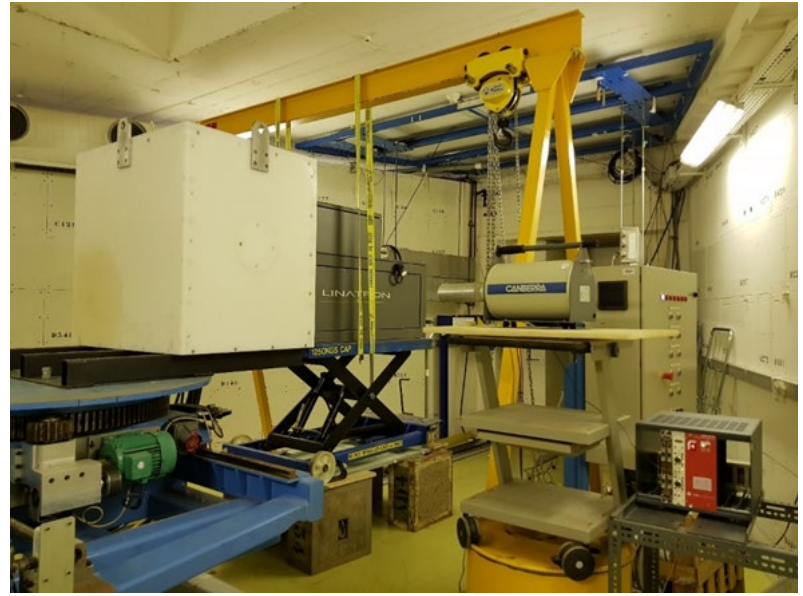

Fig. 12. View of the setup dedicated to the detection of prompt gamma-rays from neutron activation reactions.

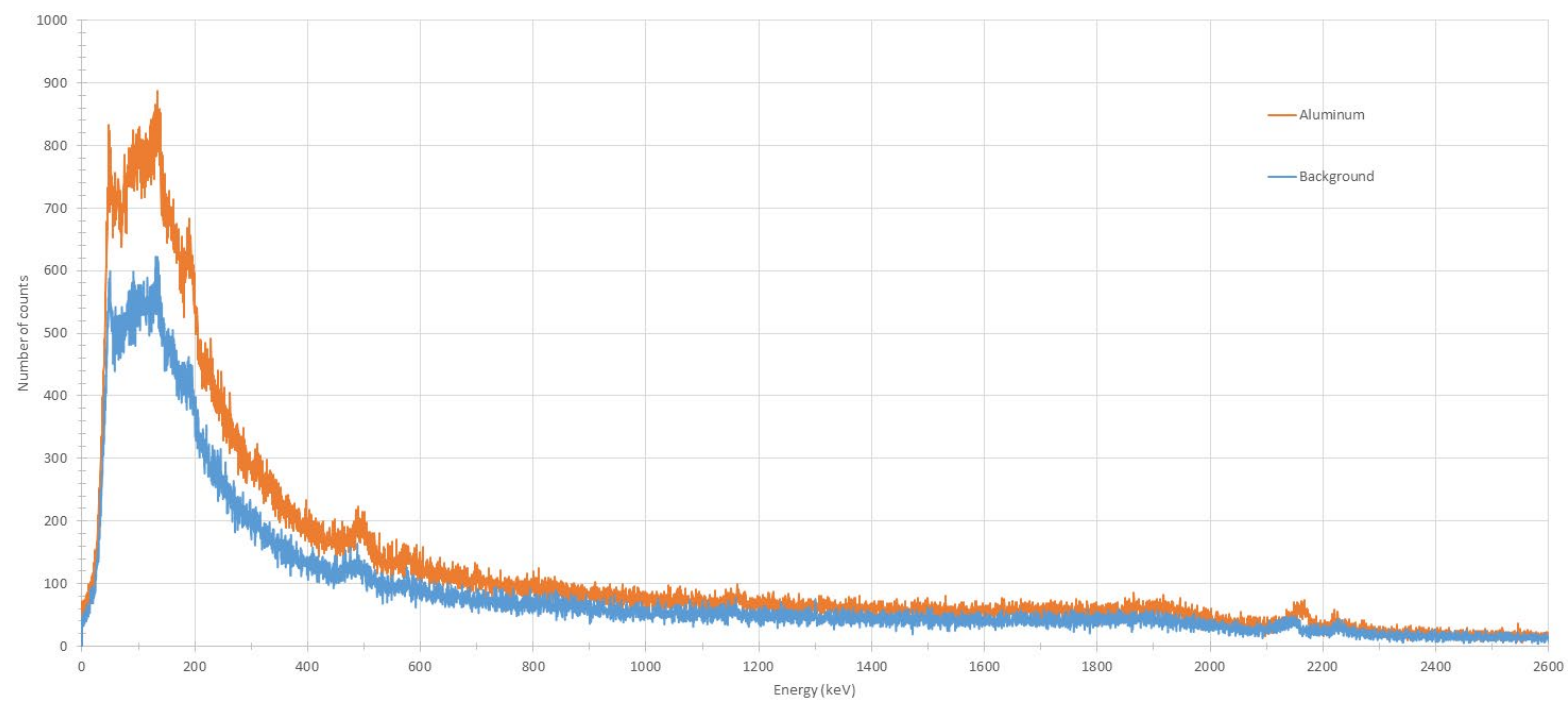

Fig. 13. Prompt gamma-ray spectrum obtained with aluminum at $6 \mathrm{MeV}$.

Fig. 12 shows a view of the experimental setup dedicated to the detection of prompt gamma-rays from neutron activation reactions. The experimental protocol was the following:

- $\quad$ Linac operated at either 6 or $9 \mathrm{MeV}$ and $40 \mathrm{~Hz}$;

- Acquisition between linac pulses during a few minutes;

- HV supply in a NIM rack located in the irradiation hall;

- Hexagon digitizer manufactured by CAEN located in the control room;

- First measurements on pure aluminum or vanadium foils of a few tens of grams.

In summary, experimental results obtained at $9 \mathrm{MeV}$ lead to failure of the HPGe detector. Nevertheless, measurements performed at $6 \mathrm{MeV}$ were successful. The inhibition signal was set at $1 \mathrm{~ms}$ and the dead time of the HPGe detector was stable and close to $70 \%$. An example of spectrum measured at $6 \mathrm{MeV}$ with a sample of aluminum is presented in Fig. 13. An excess of signal around $41 \%$ is obtained despite a low geometric detection efficiency.
Then, measurements were performed with various numbers of pure aluminum or vanadium foils. Figs. 14 and 15 present results obtained. A linear behavior can be seen between prompt gamma-ray signals and aluminum or vanadium masses. These results demonstrate the feasibility of the detection of prompt gamma-rays from neutron activation reactions by interpulse gamma spectrometry with a $6 \mathrm{MeV}$ linac and an HPGe detector.

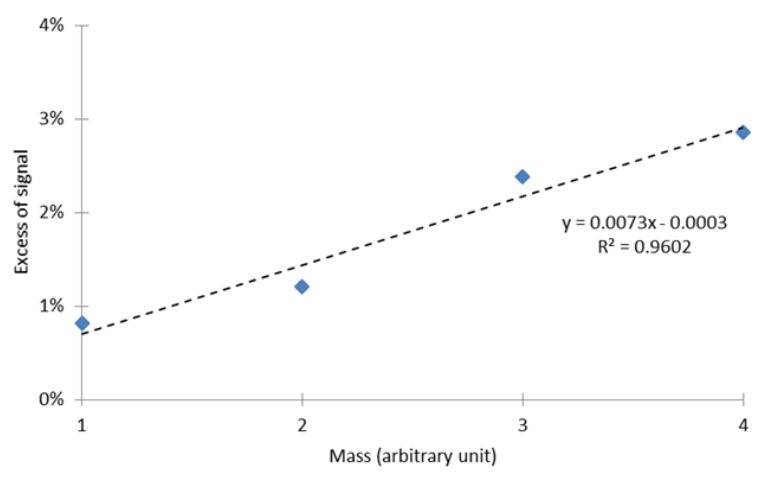

Fig. 14. Experimental results obtained for various masses of aluminum. 


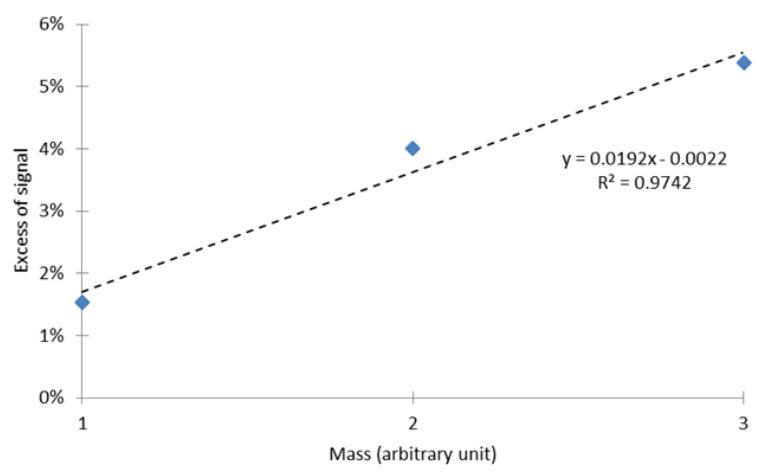

Fig. 15. Experimental results obtained for various masses of vanadium.

\section{CONCLUSION AND OUTLOOK}

Finally, photon activation with a $9 \mathrm{MeV}$ linac enabled to detect iron and silica in the samples of soil by delayed gammaray spectrometry using an HPGe detector. Using a $30 \mathrm{MeV}$ linac, much more elements would be identified in samples by photon activation. However, deployment of such a linac would not be permitted on a construction site. Neutron activation using a 6 or $9 \mathrm{MeV}$ linac and heavy water enabled to detect various elements in the samples of soil (titanium, manganese, magnesium, sodium, vanadium, and aluminum) also by delayed gamma-ray spectrometry with an HPGe detector. The feasibility of the detection of prompt gammarays from neutron activation measurements between linac pulses has been demonstrated using pure aluminum or vanadium samples.

Further, development of the interpulse gamma spectrometry technique with a linac will be pursued. A shielding of the HPGe detector will be designed, the blanking signal will be optimized, and new measurements will be performed with various materials.

\section{REFERENCES}

[1] G. Revel, « Analyse par activation », Techniques de l'Ingénieur, Réf. P2565 V3, 1999.

[2] R. M. Lindstrom, "Prompt gamma neutron activation analysis (PGAA): recent developments and applications", Journal of Radioanalytical and Nuclear Chemistry (2017) 314:843-858.

[3] C. Engelmann, « Principe, possibilités et applications de l'analyse par activation aux photons gamma ", rapport CEA, DRA/SAECNI/79187/382/CE/MC, 1979.

[4] T. Kato, I. Morita, N. Sato, "Non-destructive photon-activation analysis of standard rocks with $30 \mathrm{MeV}$ Bremsstrahlung", Journal of Radioanalytical Chemistry, Vol. 18 (1973) 97-110.

[5] A. Sari, F. Lainé, F. Carrel, "Characterization and optimization of the photoneutron flux emitted by a 6- or 9-MeV electron accelerator for neutron interrogation measurements", IEEE Trans. Nucl. Sci., Vol. 65, No. 9, 2018.

[6] A. Sari et al., "Design of a neutron interrogation cell based on an electron accelerator and performance assessment on 220 liter nuclear waste mock-up drums," IEEE Trans. Nucl. Sci., vol. 61, no. 4, pp. 2144-2148, 2014.

[7] R. Pourimani et al., "Resonance capture $\gamma$-ray spectrometry at Lead Slowing-down neutron spectrometer", Nuclear Instruments and Methods in Physics Research A 488 (2002) 226-239. 